The Development of Attention to Dynamic Facial Emotions

\author{
Alison Heck, Alyson Hock, Hannah White, Rachel Jubran, \& Ramesh S. Bhatt \\ University of Kentucky
}

Word length: 6626 (main text + references)

Correspondence:

Ramesh Bhatt

Department of Psychology

University of Kentucky

Lexington, KY 40506-0044

Phone: (859) 257-6853

e-mail: rbhatt@email.uky.edu

Author Note

This research was supported by grants from the National Science Foundation (BCS1121096) and the National Institute of Child Health and Human Development (HD075829). The authors would like to thank the infants and parents who participated in this study. Address correspondence to Ramesh S. Bhatt at rbhatt@email.uky.edu. 
Word length: 6626 (main text + references) 


\begin{abstract}
Appropriate processing of emotions is paramount for successful social functioning. Adults' enhanced attention to negative emotions like fear is thought to be a critical aspect of this adaptive functioning. Prior studies indicate that increased attention to fear relative to positive or neutral emotions begins around 7 months, and it has been suggested that this negativity bias is related to self-locomotion. However, these studies mostly used static faces, potentially limiting information available to the infant. In the current study, 3.5-month-olds $(n=24)$ and 5-month-olds $(n=24)$ were exposed to dynamic faces expressing fear, happy, or neutral emotions and a distracting peripheral checkerboard. Five-month-olds looked proportionally longer at the face compared to the checkerboard when the face was fearful than when it was happy or neutral. Conversely, 3.5month-olds did not differentiate their attention as a function of emotion. These results indicate that the onset of enhanced attention to fear occurs between 3.5 and 5 months. This finding raises questions about the developmental mechanisms that drive attentional bias, as the idea of the onset of self-locomotion being a catalyst for the development of negativity bias may no longer hold.
\end{abstract}

Keywords: emotion processing; attention; dynamic facial emotion; emotion and motor development; social perception 


\section{The Development of Attention to Dynamic Facial Emotions}

The ability to process emotions, whether in the face, voice, or body, is essential for infants' successful interactions with the environment. Individuals who experience difficulty processing emotions, such as those with autism spectrum disorder, may continue to demonstrate deficits or impairments in social functioning even into adulthood (Philip et al., 2010). However, gaps remain in our understanding of the typical progression of emotion perception during infancy. In particular, there is uncertainty about the nature of development of infants' attention to emotions. This issue is significant because it has been suggested that differential attention to emotions (e.g., enhanced attention to negative emotions like fear) has survival value and is readily seen in adults (Bannerman, Milders, de Gelder, \& Sahraie, 2009). Moreover, developmental changes in attention to emotions have been associated with critical aspects of motor development such as the onset of self-locomotion (Leppänen \& Nelson, 2009; Vaish, Grossmann, \& Woodward, 2008). However, most studies that have previously addressed this issue in infancy have used static images, which may have failed to provide a realistic picture of the development of infants' attention to emotions. In the current study, we investigated whether infants' heightened attention toward negative emotions (i.e., to fear relative to happy and neutral emotions) has a different developmental profile than previously documented when using more ecologically valid dynamic expressions of emotion.

\section{Attention to Negative Emotional Stimuli}

During the first year of life, infants appear to undergo a transition in which they switch from demonstrating heightened attention to positive emotions in their environment to instead paying greater attention to negative emotions (Vaish et al., 2008). It has been suggested that this negativity bias incurs survival value when one is faced with potentially threatening situations 
(for reviews, see Leppänen \& Nelson, 2009; Vaish et al., 2008). Many studies have examined the timing of the development of enhanced attention to threat-related negative emotions (e.g., fear, anger). For example, research by Peltola, Leppänen, and their colleagues shows that 7-montholds, but not 5-month-olds, attend more to fearful faces than to happy or neutral faces in the presence of a competing stimulus (Forssman et al., 2014; Leppänen et al., 2010; Leppänen et al., 2011; Peltola, Forssman, Puura, van IJzendoorn, \& Leppänen, 2015; Peltola, Hietanen, Forssman, \& Leppänen, 2013; Peltola, Leppänen, Palokangas, \& Hietanen, 2008; Peltola, Leppänen, Vogel-Farley, Hietanen, \& Nelson, 2009). These studies led to the conclusion that the onset of enhanced attention to negative emotions occurs around 7 months of age. Moreover, studies using threat-related but non-face stimuli like snakes (e.g., LoBue \& DeLoache, 2008) and those using social referencing paradigms that involve adults' response to novel objects with fear (e.g., Hoehl, Palumbo, Heinisch, \& Striano, 2008; Hoehl \& Striano, 2010) suggest that, starting at around 6 to 7 months, infants exhibit enhanced attention not only to negative facial emotions but also to threat-relevant objects and animals.

Although the studies outlined above have found an increase in visual attention to fear compared to happy or neutral expressions, we are aware of at least one study in which infants attended to fearful faces less than to other expressions. Serrano, Iglesias, and Loeches (1992) found that 4- to 6-month-old infants' attention to static faces expressing fear was lower compared to anger or surprise during habituation in an infant-control habituation task. However, the authors acknowledged the possibility that infants at that age may not find fear to be as familiar to them as surprise or anger. The studies reviewed above found increased attention to fear but primarily focused on infants 7 months of age and older, so the results from Serrano et al. (1992) do not necessarily contradict these findings. Moreover, it is unclear how the use of static 
faces in the Serrano et al. (1992) study, rather than realistic dynamic faces, may have affected infants' typical response to these emotions.

Moreover, in comparison to the large number of studies examining threat-related negative emotions such as fear and anger, few studies have focused on other negative emotions, such as sadness. Kahana-Kalman and Walker-Andrews (2001) found that 3.5-month-old infants were able to successfully match sad and happy faces and voices, but only when it was their own mother expressing the emotion. Additionally, infants looked longer at happy faces than at sad faces, overall. In a social referencing study, Sorce, Emde, Campos, and Klinnert (1985) found only ambiguous evidence of a response to sadness at 12 months of age. Therefore, it is unclear whether the negativity bias extends to all negative emotions, regardless of signal value, or whether it is limited to emotions that indicate threat. Thus, it is important to investigate differences within the negative emotion category. However, in the current study, we limited our scope to younger infants' attention to fear in order to clearly examine the nature of development of attention to this critical emotion in early infancy.

\section{Developmental Mechanisms}

The finding that the transition to the negativity bias begins during the second half of the first year has been thought to indicate that there are specific developmental changes occurring around this time that trigger its onset. For example, the inception of self-locomotion could lead to an increase in infants' exposure to negative emotions expressed by parents (Campos et al., 2000). An infant who is just beginning to crawl would benefit from the ability to respond to a parent's fear expression if the infant finds himself in a dangerous situation (e.g., about to crawl down the stairs). Additionally, infants begin to experience their own fear or wariness as their 
motor skills emerge (Campos, Bertenthal, \& Kermoian, 1992). Consequently, this transition to independent locomotion may trigger the onset of the negativity bias around 7 months of age.

Developmental changes in brain functions have also been associated with changes in attention to emotions. For example, Peltola, Leppänen, Mäki, and Hietanen (2009) found enhanced behavioral and physiological responses to fear at 7 months of age, but not at 5 months, and concluded that the brain network associated with the detection of emotions, particularly threat-related emotions such as fear, emerges between 5 and 7 months (for a review, see Leppänen \& Nelson, 2009). Peltola, Leppänen, Mäki, and colleagues (2009) also suggested that 5-month-olds did not show differential attention to fear and happy faces because they do not yet understand the signal value of threat-related emotions. However, the authors also acknowledged that one limitation of their study was the use of static photographs and that infants have shown sensitivity to dynamic emotions at an earlier age than seen with static expressions.

\section{Static versus Dynamic Emotion Stimuli}

The majority of studies on infants' processing of negative facial emotions have used static images of emotion faces (e.g., Forssman et al., 2014; Leppänen et al., 2010; Leppänen, et al., 2011; Peltola et al., 2015; Peltola et al., 2013; Peltola et al., 2008; Peltola, Leppänen, VogelFarley, et al., 2009). However, emotions are by their very nature dynamic (Caron, Caron, \& Myers, 1985); therefore, static displays of emotions sacrifice ecological validity. In fact, Caron et al. (1985) concluded that even 7- to 9.5-month-olds are unable to discriminate between static happy and angry faces when salient features (e.g., toothiness) are controlled for, thus supporting their conclusion that infants are not yet capable of extracting emotion information from static faces. Consequently, when using static faces to study emotion processing, we may be underestimating infants' abilities. 
Across many research domains, it has been found that infants show improved performance when tested with dynamic stimuli (e.g., Bahrick, Lickliter, \& Flom, 2004; Caron et al., 1985; Quinn et al., 2011; Walker-Andrews, 1997). For example, infants look longer at internal features of the face (as opposed to the edges) at an earlier age when faces are dynamic than when they are static (Haith, Bergman, \& Moore, 1977). Additionally, 5-month-old infants look longer overall at dynamic emotion faces compared to static faces (Wilcox \& Clayton, 1968). Montague and Walker-Andrews (2001), using a live peekaboo paradigm, found that 4month-olds are sensitive to fear and anger in addition to sadness and happiness. Thus, this study provided additional evidence that using more realistic displays of emotion can demonstrate infants' sensitivity to different emotions at an earlier age than when using static stimuli.

Moreover, some researchers have suggested that the changes in expression portrayed in dynamic faces might be inherently more informative than static information. For example, a video beginning with a neutral face and steadily changing to portray increasingly more emotion until the target intensity is reached is likely to be more informative than a series of discrete static images that provide the same amount of information. Empirical support for this possibility was provided by Ambadar, Schooler, and Cohn (2005), who found that adults are more confident in their emotion ratings of subtle expressions in dynamic than in static displays. Ambadar et al. (2005) ruled out the possibilities that temporal characteristics or increased information (e.g., more samples) were driving the benefits of dynamic displays. They concluded that movement per se enhances adults' sensitivity to changes in facial expressions, which helps them to correctly identify the subtle emotion being addressed. This study in particular underscores the importance of using dynamic facial displays of emotion to improve the ecological validity of research on emotion processing. 


\section{Current Study}

We examined young infants' attention to fearful emotions by documenting whether dynamic fearful faces hold infants' attention longer than other emotions in the presence of competition from a peripheral stimulus. We utilized the attention overlap task used by Peltola, Leppänen, and their colleagues (Forssman et al., 2014; Leppänen et al., 2010; Leppänen et al., 2011; Peltola et al., 2015; Peltola et al., 2013; Peltola et al. 2008; Peltola, Leppänen, VogelFarley, et al., 2009). In this task, infants are shown a centrally presented static face for a specified amount of time. Then, while the face remains on the screen, a peripheral stimulus (e.g., a checkerboard) is presented on the left or right of the screen and both stimuli stay on the screen for the remainder of the trial. The question of interest is whether infants look longer at the fearful faces than at the happy and neutral faces in the presence of the peripheral stimulus. As described earlier, previous studies indicate that static fearful faces hold infants' attention longer relative to other emotions (i.e., happy, neutral) at 7 months but not at 5 months. However, the determination that the onset of the negativity bias occurs around 7 months may not be accurate given that the studies that led to this conclusion mostly used static stimuli. To address this possibility, the current study tested 3.5- and 5-month-old infants on more realistic, dynamic displays of actresses expressing fear, happy, and neutral emotions.

If both 3.5- and 5-month-olds show heightened attention to a dynamic fearful face, then this would indicate that even by 3.5 months of age infants attend to emotions appropriately. Since there is some evidence that newborns show a positivity bias for happy faces (Farroni, Menon, Rigato, \& Johnson, 2007), this would indicate that the negativity bias develops sometime during the first three months. However, if only 5-month-olds exhibit increased attention to dynamic fearful faces, then the transition occurs sometime between 3.5 and 5 months 
of age. Alternatively, if the transition to heightened attention for fear is not seen until after 5 months of age, when certain experiential factors come into play (e.g., self-locomotion), then neither 3.5-months-olds nor 5-month-olds should exhibit negativity bias even with dynamic videos. Being able to detect when this transition occurs with dynamic stimuli would in turn allow for more precise identification of the mechanisms involved in the development of infants' emotion perception.

\section{Method}

\section{Participants}

The final sample included 24 3.5-month-olds ( $M$ age $=105.42$ days, $S D=8.93 ; 15$ girls $)$ and 24 5-month-olds ( $M$ age $=150.67$ days, $S D=6.88 ; 12$ girls), who were recruited through birth announcements and from a local hospital. The participants were primarily Caucasians from middle-class families. Two additional 3.5-month-olds were excluded due to insufficient data $(<$ $20 \%$ of trials with valid data) and for not completing the task due to fussiness. One additional 5month-old was also not included due to insufficient data.

\section{Stimuli and Apparatus}

The stimuli used were validated dynamic videos of three Caucasian females silently depicting fear, happy, and neutral emotion expressions while facing forward. One exemplar per emotion for each female was selected from the Amsterdam Dynamic Facial Expression Set (ADFES; Van der Schalk, Hawk, Fischer, \& Doojse, 2011). Infants in both age groups were randomly assigned to one of three female models (models F01, F03, or F05 from the ADFES). For happy and fear expressions, each model initially began with a neutral expression and after approximately $500 \mathrm{~ms}$ shifted to the target emotion expression and held it for approximately $5000 \mathrm{~ms}$. For the neutral expression, the initial expression was maintained through the length of 
the video, but head movements and eye blinking preserved the dynamic nature of the expression. Arousal ratings provided in Van der Schalk et al. (2011) indicate that the happy stimuli were rated higher in arousal $(M=5.27, S D=0.67)$ than fearful faces $(M=3.87, S D=1.10)$. Therefore, if the results were consistent with the prediction that fearful faces would hold attention more than happy and neutral faces, they cannot be due to a preference for the more intense stimuli because the fearful stimuli were less intense than the happy stimuli.

Unfortunately, arousal ratings for the neutral stimuli are not available (Van der Schalk et al., 2011).

The peripheral stimulus was a black and white checkerboard that appeared on the left or right side of the screen halfway through each 6000-ms long trial (i.e., at $3000 \mathrm{~ms}$ ). The side of presentation of the checkerboard was counterbalanced across trials. Once displayed on the screen, the checkerboard flickered at a rate of $10 \mathrm{~Hz}$ for the first $1000 \mathrm{~ms}$ and stayed stationary for the remaining 2000 ms (as in Peltola, Leppänen, Vogel-Farley et al., 2009).

For a given infant, each emotion exemplar (fear, happy, and neutral) from one of the female models was repeated 12 times, for a total of 36 trials. There were four different random presentation orders for each model, counterbalanced for order of emotion presentation and the left/right location of the checkerboard, with the restriction that no emotion was presented more than twice in a row. Before each trial, an attention-getter consisting of alternating green and purple shapes was presented in the center of the screen (see Figure 1).

Gaze patterns were recorded using a Tobii TX300 binocular near-infrared eye-tracking system, which has a 23-inch TFT monitor. This system has a precision rate of $0.15^{\circ}$ and accuracy rate of $0.5^{\circ}$ when the participant is seated $55-60 \mathrm{~cm}$ from screen. The sampling rate was $300 \mathrm{~Hz}$. All stimuli were presented in Tobii Studio v.3.2.1 through an external program using the screen 
recording feature. The data were filtered using the Tobii Studio I-VT fixation filter, which classifies fixations as any looks that exceed $60 \mathrm{~ms}$ in duration while remaining within a $0.5^{\circ}$ radius. The Tobii TX300 eye-tracker is capable of compensating for robust head movements and takes approximately $100 \mathrm{~ms}$ to recover tracking ability after movement has occurred.

Additionally, if one eye is unable to be tracked at a given time frame, data from the other eye is used to determine the gaze coordinates.

Areas of interest (AOIs) for the face and the peripheral stimulus were drawn for each trial using Tobii Studio. The size and shape of the AOIs was the same for all emotions and actresses. The face AOI was a square box drawn around the entire head and upper shoulders of the actress, which subtended a vertical and horizontal visual angle of $10.88^{\circ}$ from the viewer. The peripheral stimulus AOI was a rectangular box drawn around the checkerboard, which subtended vertical and horizontal visual angles of $12.08^{\circ}$ and $4.85^{\circ}$, respectively.

\section{Procedure}

Infants sat on their parent's lap in a darkened chamber approximately $60 \mathrm{~cm}$ in front of the eye-tracking monitor. The parents wore opaque glasses that prevented them from seeing the images. Initially, a five-point infant calibration procedure, in which an animated, colorful object paired with an engaging sound sequentially appeared in the four corners and center of the screen, was used to calibrate the eye-tracker. When the experimenter determined that the infant had fixated on a particular target by using the live viewer tool in Tobii Studio, a key was pressed to advance to the next target until it had been shown in all five locations. This process was repeated until there was minimal foveal spread from each of the five target points for both eyes.

Following successful calibration, the stimuli were presented in an attention overlap task as closely as possible to Peltola et al. (2008; see Figure 1 for a schematic of the overlap task used 
in the current study). Each trial began with the attention-getter (alternating colorful shapes)

presented in the center of the computer monitor. Once the infants focused on the attention-getter, it was removed and an emotion video (fear, happy, or neutral) was displayed in the center of the screen for $3000 \mathrm{~ms}$. Then, while the face remained on the screen, the peripheral stimulus (a black and white checkerboard) was presented on either the left or right side of the screen. Both the dynamic face and checkerboard were visible for an additional $3000 \mathrm{~ms}$, after which the attentiongetter was again displayed on the screen. This process was repeated for each of the 36 trials.

(a)

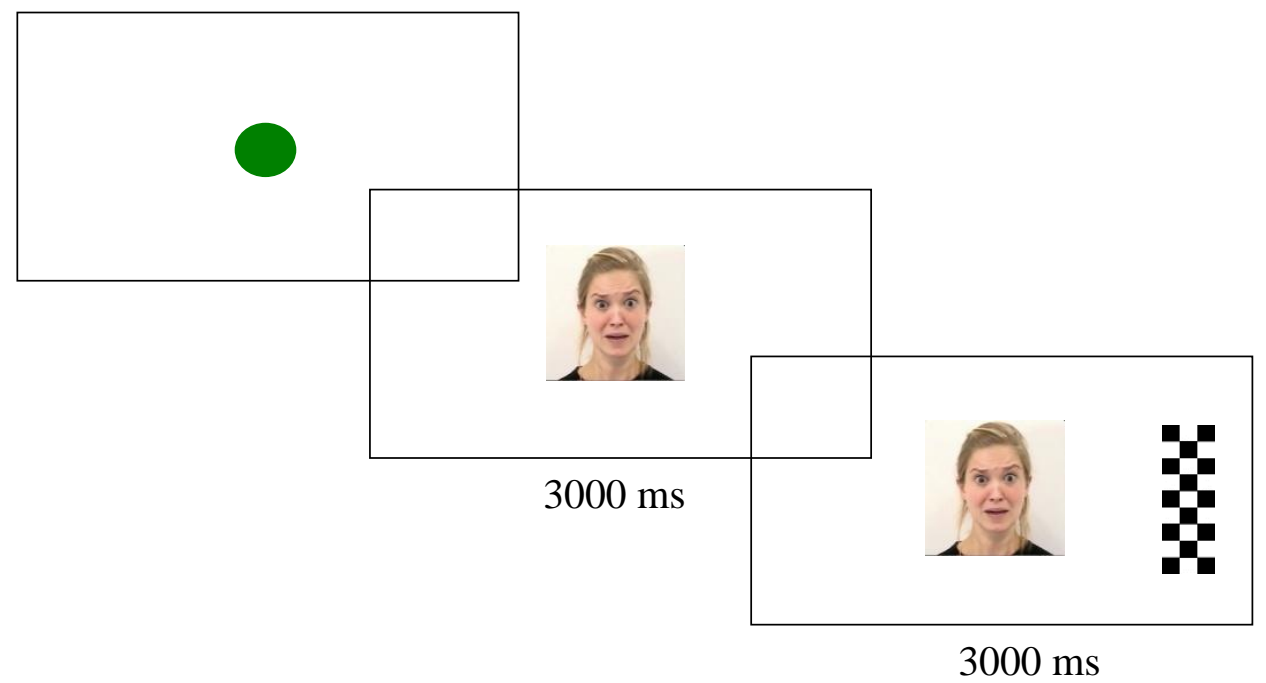

(b)

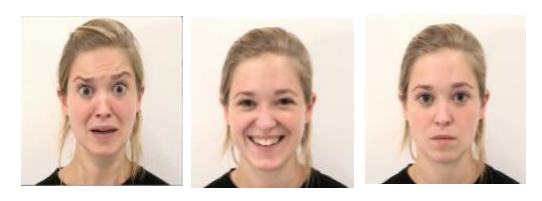

Figure 1. Schematic of the attention overlap task (a) and screenshots portraying examples of the three emotions used in this study: fear, happy, and neutral (b). At the beginning of each trial, an animated attention-getter was presented. Once the infant fixated the attention-getter, a dynamic female face depicting one of three emotions was presented in the center of the screen for 3000 ms. Then, the checkerboard appeared on the left or right side of the screen while the face continued to be visible, and both remained on the screen for the final $3000 \mathrm{~ms}$ of the trial. 


\section{Results}

The primary question addressed in this study was whether 3.5- and 5-month-olds' attention would be engaged to a greater extent by dynamic fearful faces compared to dynamic happy or neutral expressions in the presence of a competing stimulus (i.e., the flashing checkerboard). Therefore, we analyzed looking behavior during the final $3000 \mathrm{~ms}$ of each trial, during which both the emotion face and checkerboard pattern were displayed. Specifically, we examined infants' proportional look durations to the face relative to the checkerboard (i.e., total fixation duration to the face divided by total fixation duration to the face + checkerboard, and the ratio multiplied by 100) across trials for each emotion. If this proportion was larger during fear trials compared to happy and neutral trials, then it would indicate that fear holds infants' attention more than happy and neutral emotions.

Individual trials were excluded if there were no looks to the screen during the trial, if there were anticipatory looks to the peripheral stimulus location before the stimulus appeared or within $180 \mathrm{~ms}$ of stimulus onset, or if the infant looked at the stimulus without first fixating on the face. These exclusion criteria were based on criteria used in attention overlap tasks by Leppänen, Peltola and colleagues (Forssman et al., 2014; Leppänen et al., 2010; Leppänen et al., 2011; Peltola et al., 2015; Peltola et al., 2013; Peltola, Leppänen, \& Hietanen, 2011; Peltola et al., 2008). On average, 4.33 trials $(S E=0.54)$ were excluded per participant, and the number of trials excluded did not differ statistically as a function of emotion or age. Recall that four quasirandom trial orders and three different face models were used in this experiment. Preliminary analyses failed to reveal any effects of trial order or model, so the following analyses were collapsed across these factors. 
The data are presented in Figure 2. An emotion (fear, happy, neutral) x age (3.5 months, 5 months) mixed ANOVA revealed that the main effect of age was not significant $[F(1,46)=$ $\left.\left.3.50, p=.07, \eta_{\mathrm{p}}{ }^{2}=.07\right)\right]$. However, there was a significant main effect of emotion $[F(2,92)=$ $\left.13.40, p<.001, \eta_{\mathrm{p}}^{2}=.23\right]$, which was qualified by a significant emotion $\mathrm{x}$ age interaction $[F(2$, $\left.92)=3.52, p=.03, \eta_{\mathrm{p}}{ }^{2}=.07\right]$. To investigate this interaction, the data from each age group were examined separately. Three-and-a-half-month-olds' proportional look durations did not differ significantly as a function of emotion $\left[F(2,46)=1.78, p=.18, \eta_{\mathrm{p}}{ }^{2}=.07\right]$. In contrast, emotion significantly affected 5-month-olds' performance $\left[F(2,46)=14.40, p<.001, \eta_{\mathrm{p}}{ }^{2}=.39\right.$; see Figure 2]. Pairwise comparisons of 5-month-olds' data using a Bonferroni correction (adjusted $\alpha$ $=.0167$ ) revealed that infants fixated the face relative to the checkerboard more during fear trials $(M=67.92 \%)$ than during both happy $[M=60.41 \% ; t(23)=3.80, p=.001, d=0.47]$ and neutral trials $[M=56.57 \% ; t(23)=4.67, p<.001, d=0.67]$. The difference in proportion fixation to the face in happy versus neutral trials was not significant $[t(23)=-1.91, p=.07, d=0.23]$. These results indicate that fearful faces hold 5-month-olds' attention more than happy or neutral faces. However, 3.5-month-olds do not show such evidence of a negativity bias. 


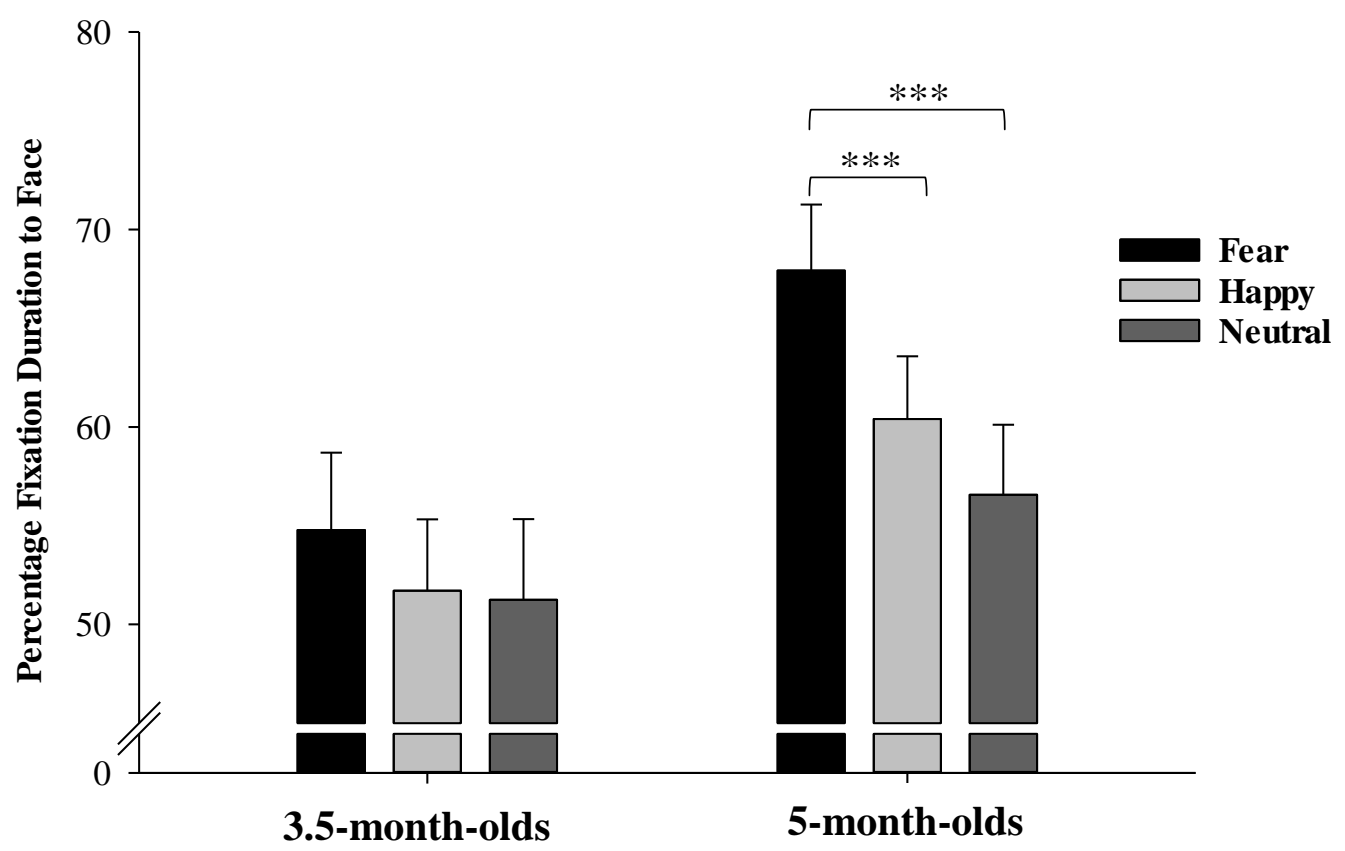

Figure 2. Percentage of total fixation duration to the face relative to the peripheral target as a function of emotion and age group. Five-month-olds looked significantly longer at fearful faces relative to the checkerboard compared to both happy and neutral faces; 3-month-olds did not significantly differentiate their attention as a function of emotion. The error bars indicate $1 \mathrm{SE}$. Note: $* * * p<.001$

In order to examine any potential influence from the infants' looking prior to the onset of the peripheral stimulus, the average fixation duration on the face during the first $3000 \mathrm{~ms}$ of each trial was analyzed using an emotion (fear, happy, neutral) x age (3.5 months, 5 months) mixed ANOVA. The main effects of emotion $\left[F(2,92)=1.81, p=.17, \eta_{\mathrm{p}}{ }^{2}=.04\right]$ and age $[F(1,46)=$ $\left.1.20, p=.30, \eta_{\mathrm{p}}{ }^{2}=.02\right]$ as well as the emotion $\mathrm{x}$ age interaction were not significant $[F(2,92)=$ $\left.0.68, p=.51, \eta_{\mathrm{p}}{ }^{2}=.02\right]$, indicating no differences in look duration to the face stimuli before the onset of the peripheral stimulus (see Table 1). Therefore, differences in attention during the 
overlap period were not due to differences in look durations prior to the onset of the peripheral stimulus.

Table 1

Mean Pre-Distracter Fixation Duration and Mean Latency to Fixate on Peripheral Distracter

\begin{tabular}{|c|c|c|}
\hline & $\begin{array}{c}\text { 3.5-month-olds }(n=24) \\
M(S E)\end{array}$ & $\begin{array}{c}\text { 5-month-olds }(n=24) \\
M(S E)\end{array}$ \\
\hline \multicolumn{3}{|c|}{ Face Fixation Duration (ms) } \\
\hline Fear & 1863.65 (149.34) & $2117.10(111.46)$ \\
\hline Happy & $1909.52(150.01)$ & $2080.10(110.96)$ \\
\hline Neutral & $1840.41(160.63)$ & 1994.83 (121.13) \\
\hline \multicolumn{3}{|c|}{$\underline{\text { Latency }(\mathrm{ms})}$} \\
\hline Fear & $1034.83(48.93)$ & $856.26(51.28)$ \\
\hline Happy & $1086.98(40.41)$ & 811.09 (57.28) \\
\hline Neutral & $1102.70(42.63)$ & $811.45(47.25)$ \\
\hline
\end{tabular}

Finally, we tested for effects of emotion on the latency to fixate on the checkerboard in each trial (see Table 1). The latency measure was averaged across the trials in which there was a fixation on the checkerboard. However, an emotion (fear, happy, sad) x age (3.5 months, 5 months) mixed ANOVA did not reveal a significant main effect of emotion $[F(2,92)=0.04, p=$ $\left..96, \eta_{\mathrm{p}}^{2}=.001\right]$ or a significant emotion $\mathrm{x}$ age interaction $\left[F(2,92)=1.11, p=.33, \eta_{\mathrm{p}}{ }^{2}=.02\right]$. There was a significant effect of age $\left[F(1,46)=25.48, p<.001, \eta_{\mathrm{p}}{ }^{2}=.36\right]$, in that the 5-montholds overall had significantly shorter latencies $[M=826.27 \mathrm{~ms}, S E=38.43]$ to fixate on the 
checkerboard than the 3.5-month-olds $[M=1074.84 \mathrm{~ms}, S E=150.86]$, but this did not vary by emotion.

The fact that the latency measure did not reveal differential attention to emotions even in older infants is not surprising given that this measure has been found to be inconsistent in prior studies. While a few studies have found that 7-month-olds take longer to attend to the peripheral stimulus in the presence of fearful faces than in the presence of happy and neutral faces (Leppänen et al., 2010; Peltola, Leppänen, Hietanen, et al., 2009; Peltola et al., 2011), many more studies have failed to report such latency results while indicating differential attention to fearful faces using other measures (e.g., Forssman et al., 2014; Leppänen et al., 2011; Nakagawa \& Sukigara, 2012; Peltola et al., 2008; Peltola et al., 2013; Peltola et al., 2015). Given this fact, we believe that it is more prudent to rely upon a direct measure of attention holding, namely the duration of looking to the face stimulus in the presence of the distracter. As described earlier, this measure clearly indicates that 5-month-olds in this experiment, but not 3.5-month-olds, looked longer at fearful than at happy and neutral faces in the presence of the checkerboard (Figure 2). Thus, the current study, which used dynamic stimuli, found enhanced attention to negative emotions (specifically fear) at a younger age than in prior studies. However, 3.5-month-olds failed to exhibit a similar pattern of looking, suggesting that enhanced attention to negative stimuli develops between 3.5 and 5 months of age.

\section{Discussion}

The primary goal of this study was to investigate whether the attentional bias to fearful faces previously documented in infants at 7 months of age (e.g., Peltola et al., 2008) could be found at 3.5 and 5 months if more ecologically valid dynamic videos, instead of static images, were used. Five-month-olds exhibited increased attention to dynamic fearful faces relative to a 
peripheral checkerboard pattern compared to both happy and neutral faces, whereas 3.5-montholds failed to respond differentially to the three emotions. Therefore, the transition towards increased attention to negative emotions occurs sometime between 3.5 and 5 months of age rather than between 5 and 7 months as suggested in prior studies. This change in the timing of differential attention to emotions has implications for models of the association between the development of attention to emotions and motor development, specifically self-locomotion.

The present finding that 5-month-olds demonstrate an attentional bias for fear differs from that of Peltola, Leppänen, Mäki et al. (2009), who found differential attention at 7 but not at 5 months. A main methodological difference was the use of dynamic emotion displays rather than static images. As previously discussed, few studies have employed dynamic faces in their investigations of infants' processing of emotions. However, dynamic stimuli may be more realistic and beneficial for information processing in comparison to static stimuli (Quinn et al., 2011). Therefore, we hypothesized that the use of dynamic emotion faces would lead to evidence that enhanced attention to fear occurs at an earlier age than previously reported. This prediction was supported by the performance of 5-month-olds in the current study, which suggests that features specific to dynamic stimuli may have led to this enhanced attention for fear even in young infants.

Quinn et al. (2011) noted that differences seen in studies of emotion processing between those using dynamic versus static stimuli may be unrelated to the emotional content of the faces per se. They argued that tests using dynamic displays generally tend to be longer than those using static displays. Although our study was patterned after those by Peltola and colleagues, our trials were longer (6000 ms compared to 4000 ms in Peltola, Leppänen, Vogel-Farley et al., 2009), but the overlap period when the face and checkerboard were both on the screen and was 
the basis for the main analyses was identical (3000 ms). Additionally, even prior studies in which infants experienced long durations of exposure to static emotion stimuli failed to find enhanced attention to fear stimuli at 5 months. For example, Peltola, Leppänen, Mäki et al. (2009) found differential responses to emotions only at 7 months and not at 5 months even though they used 10-s long visual paired comparison trials to document visual scanning and 150 trials of happy and fearful faces presented for $1000 \mathrm{~ms}$ each in the event-related potential portion of the study. Therefore, it is unlikely that additional time is the explanation for the differences seen in our study compared to others using static images. However, there are other ways that dynamic stimuli may have benefited the 5-month-olds in this study.

For example, Longhi et al. (2015) found that newborns distinguish between possible and impossible hand gestures only when hands were dynamic, not static. The authors speculate that movement provides additional information highlighting the novelty of the appearance of the impossible hand gesture compared to the possible one. In contrast, static images are unable to convey enough information for infants to discern the novelty of the impossible hand pose. Interestingly, a similar novelty-based account of the negativity bias in emotion processing has been proposed, namely that the enhanced responses to fear and anger are due to a novelty effect, based on the assumption that young infants are not exposed to negative emotions as much as to positive emotions. Peltola et al. (2008) tested this novelty effect argument with 7-month-olds using static fear, happy, and novel (female face with cheeks puffed out) facial expressions and a control stimulus (scrambled face). Infants in that study demonstrated enhanced attention only to fearful faces and not to other novel faces, which lessens the likelihood that novelty is the primary reason for the heightened attention to fear. Moreover the argument that novelty drove 5-montholds' but not 3.5-month-olds' enhanced attention to fear in the current study is rendered unlikely 
given the plausible assumption that 3.5-month-old infants would have had even less exposure to fear and therefore would be even more likely to show a novelty effect compared to 5-month-old infants.

An alternate explanation for the findings in the current study is that 5-month-olds have learned the signal value of emotional faces at least to some extent, leading them to maintain their attention longer when socially-relevant information, especially threat-related information, is available to them. In contrast, 3.5-month-olds may not yet understand the importance of the emotions being expressed, which may have resulted in their failure to differentially attend to fear.

The idea that 5-month-olds are sensitive to the social significance of fear is inconsistent with the hypotheses that negativity bias is related to the onset of self-locomotion (e.g., crawling; see Vaish et al., 2008). Although locomotor experience was not explicitly measured in this study, it is unlikely that infants at either age in the current study had attained independent crawling or walking experience. However, the onset of visually-directed reaching behaviors around 4-5 months (Thelen \& Spencer, 1998) may have been a sufficient catalyst for changes in emotion processing. Once infants begin to reach for objects on their own, it is possible that parents start reacting with increased negative emotions, especially if the infant picks up an object that is potentially dangerous or unsanitary. In this sense, the previous explanations given for why infants experience an increase in exposure to negative emotions at the onset of crawling may hold for this motor milestone as well.

The possibility that reaching behavior is correlated with developmental changes in social perception is supported by prior research. Libertus and Needham (2011) examined how 3- and 5month-old infants" active exploration of objects relates to their face perception by using a "sticky 
mittens" procedure. One group of 3-month-olds was given experience handling objects with the assistance of Velcro® gloves, while another group received passive contact with the object by the parent touching it to the glove. Infants in these two groups were given similar amounts of exposure to the objects, and matched in terms of sitting experience, and parent engagement. However, only 3-month-olds with successful independent grasping experience demonstrated a preference for human faces over colorful toys, whereas those in the passive exploration condition and a third untrained control condition did not. In other words, infants who had experience with actively exploring their environment using reaching behaviors seemed to be more aware of the importance of faces in their environment. Libertus and Needham (2011) concluded that development in motor skills, such as reaching, is associated with development in the social domain. It is thus possible that differential attention to particular emotions is similarly correlated with motor development such as reaching behavior between 3.5 and 5 months of age. Examining whether experience with independent reaching at 3.5 months leads to a shift in attention to fearful faces similar to 5-month-olds would be a test of this hypothesis.

The relationship between motor milestones like reaching and emotion processing is also supported by the suggestion that triadic interactions involving two individuals and an object early in infancy are a precursor to the development of joint attention skills (Striano \& Reid, 2006). As discussed earlier, social referencing studies show that infants are responsive to positive and negative emotion expressions directed towards objects (Hoehl et al., 2008; Hoehl \& Striano, 2010). Therefore, the rise in the frequency of parent/infant/object interactions involving infants' reaching for and playing with objects may increase infants' exposure to varied emotions by their caregivers, specifically negative emotions, which in turn may lead older and more experienced infants to attend differentially to various emotions. 
In summary, the findings from the current study make an important contribution to the understanding of the development of increased attention to negative emotions, particularly fear. Whereas previous studies using static images found that this shift appears between 5 and 7 months of age, the results from this study using dynamic emotion displays indicate that the transition actually occurs sometime between 3.5 and 5 months of age. This change in the timing of the transition opens up questions about previously proposed associations between specific aspects of motor development, specifically self-locomotion, and emotion processing. 


\section{References}

Ambadar, Z., Schooler, J.W., \& Cohn, J.F. (2005). Deciphering the enigmatic face: The importance of facial dynamics in interpreting subtle facial expressions. Psychological Science, 16, 403-410. DOI: 10.1111/j.0956-7976.2005.01548.x

Bannerman, R. L., Milders, M., de Gelder, B., \& Sahraie, A. (2009). Orienting to threat: Faster localization of fearful facial expressions and body postures revealed by saccadic eye movements. Proceedings of the Royal Society of London B: Biological Sciences, 276(1662), 1635-1641. DOI:10.1098/rspb.2008.1744

Bahrick, L.E., Lickliter, R., \& Flom, R. (2004). Intersensory redundancy guides the development of selective attention, perception, and cognition in infancy. Current Directions in Psychological Science, 13, 99-102. DOI: 10.1111/j.0963-7214.2004.00283.x

Campos, J.J., Anderson, D.I., Barbu-Roth, M.A., Hubbard, E.M., Hertenstein, M.J., \& Witherington, D. (2000). Travel broadens the mind. Infancy, 1, 149-219. DOI: 10.1207/S15327078IN0102_1

Campos, J.J., Bertenthal, B.I., \& Kermoian, R. (1992). Early experience and emotional development: The emergence of wariness of heights. Psychological Science, 3, 61-64. DOI: $10.1111 / j .1467-9280.1992 . t b 00259 . x$

Caron, R.F., Caron, A.J., \& Myers, R.S. (1985). Do infants see emotional expressions in static faces? Child Development, 56, 1552-1560. DOI: http://www.jstor.org/stable/1130474

Farroni, T., Menon, E., Rigato, S., \& Johnson, M.H. (2007). The perception of facial expressions in newborns. European Journal of Developmental Psychology, 4, 2-13. DOI: $10.1080 / 17405620601046832$

Forssman, L., Peltola, M.J., Yrttiaho, S., Puura, K., Mononen, N., Lehtimä, T., \& Leppänen, J.M. 
(2014). Regulatory variant of the TPH2 gene and early life stress are associated with heightened attention to social signals of fear in infants. Journal of Child Psychology and Psychiatry, 55, 793-801. DOI:10.1111/jcpp.12181

Haith, M.M., Bergman, T., \& Moore, M.J. (1977). Eye contact and face scanning in early infancy. Science, 198, 853-855. DOI: http://dx.doi.org/10.1126/science.918670

Hoehl, S., Palumbo, L., Heinisch, C., \& Striano, T. (2008). Infants' attention is biased by emotional expressions and eye gaze direction. Developmental Neuroscience, 19, 579-582. DOI: 10.1097/WNR.0b013e3282f97897

Hoehl, S., \& Striano, T. (2010). The development of emotional face and eye gaze processing. Developmental Science, 13, 813-825. DOI: 10.1111/j.1467-7687.2009.00944.x

Kahana-Kalman, R., \& Walker-Andrews, A.S. (2001). The role of person familiarity in young infants' perception of emotional expressions. Child Development, 72, 352-369. DOI: $10.1111 / 1467-8624.00283$

Leppänen, J., \& Nelson, C.A. (2009). Tuning the developing brain to social signals of emotions. Nature Reviews Neuroscience, 10, 37-47. DOI: 10.1038/nrn2554

Leppänen, J., Peltola, M.J., Mäntymaa, M., Koivuluoma, M., Salminen, A., \& Puura, K. (2010). Cardiac and behavioral evidence for emotional influences on attention in 7-month-old infants. International Journal of Behavioral Development, 34, 547-553, DOI: $10.1177 / 0165025410365804$

Leppänen, J., Peltola, M.J., Puura, K., Mäntymaa, M., Mononen, N., \& Lehtimäki, T. (2011). Serotonin and early cognitive development: Variation in the tryptophan hydroxylase 2 gene is associated with visual attention in 7-month-old infants. Journal of Child Psychology and Psychiatry, 52, 1144-1152. DOI:10.1111/j.1469-7610.2011.02391.x 
Libertus, K., \& Needham, A. (2011). Reaching experience increases face preference in 3-monthold infants. Developmental Science, 14, 1355-1364. DOI: 10.1111/j.14677687.2011.01084.x

LoBue, V., \& DeLoache, J.S. (2010). Superior detection of threat-relevant stimuli in infancy. Developmental Science, 13, 221-228. DOI: 10.1111/j.1467-7687.2009.00872.x

Longhi, E., Senna, I., Bolognini, N., Bulf, H., Tagliabue, P., Macchi Cassia, V., \& Turati, C. (2015). Discrimination of biomechanically possible and impossible hand movements at birth. Child Development, 86, 632-641. DOI: 10.1111/cdev.12329

Montague, D.P.F., \& Walker-Andrews, A.S. (2001). Peekaboo: A new look at infants' perception of emotion expressions. Developmental Psychology, 37, 826-838. DOI: $10.1037 / / 0012-1649.37 .6 .826$

Nakagawa, A., \& Sukigara, M. (2012). Difficulty in disengaging from threat and temperamental negative affectivity in early life: A longitudinal study of infants ages 12-36 months. Behavioral and Brain Functions, 8(40), 1-8. DOI: 10.1186/1744-9081-8-40

Peltola, M.J., Forssman, L., Puura, K., van IJzendoorn, M.H., \& Leppänen, J.M. (2015). Attention to faces expressing negative emotion at 7 months predicts attachment security at 14 months. Child Development, 86, 1321-1332. DOI: 10.1111/cdev.12380

Peltola, M.J., Hietanen, J.K., Forssman, L., \& Leppänen, J.M. (2013). The emergence and stability of the attentional bias to fearful faces in infancy. Infancy, 18, 905-926. DOI: 10.1111/infa.12013

Peltola, M.J., Leppänen, J.M., \& Hietanen, J.K. (2011). Enhanced cardiac and attentional responding to fearful faces in 7-month-old infants. Psychophysiology, 48, 1291-1298. DOI: 10.1111/j.1469-8986.2011.01188.x 
Peltola, M.J., Leppänen, J.M., Mäki, S., \& Hietanen, J.K. (2009). Emergence of enhanced attention to fearful faces between 5 and 7 months of age. Social Cognitive and Affective Neuroscience, 4, 134-142. DOI:10.1093/scan/nsn046

Peltola, M.J., Leppänen, J.M., Palokangas, T., \& Hietanen, J.K. (2008). Fearful faces modulate looking duration and attention disengagement in 7-month-old infants. Developmental Science, 11, 60-68. DOI: 10.1111/j.1467-7687.2007.00659.x

Peltola, M.J., Leppänen, J.M., Vogel-Farley, V.K., Hietanen, J.K., \& Nelson, C.A. (2009). Fearful faces but not fearful eyes alone delay attention disengagement in 7-month-old infants. Emotion, 9, 560-565. DOI: 10.1037/a0015806

Philip, R.C.M., Whalley, H.C., Stanfield, A.C., Sprengelmeyer, R., Santos, I.M., Young, A.W., Atkinson, A.P., ... Hall, J. (2010). Deficits in facial, body movement and vocal emotional processing in autism spectrum disorders. Psychological Medicine, 40, 1919-1929. DOI: $10.1017 / \mathrm{S} 0033291709992364$

Quinn, P.C., Anzures, G., Izard, C.E., Lee, K., Pascalis, O., Slater, A.M., \& Tanaka, J.W. (2011). Looking across domains to understand infant representation of emotion. Emotion Review, 3, 197-206. DOI: 10.1177/1754073910387941

Serrano, J.M., Iglesias, J., \& Loeches, A. (1992). Visual discrimination and recognition of facial expressions of anger, fear, and surprise in 4- to 6-month-old infants. Developmental Psychobiology, 25, 411-425. DOI: 10.1002/dev.420250603

Sorce, J.F., Emde, R.N., Campos, J., \& Klinnert, M.D. (1985). Maternal emotional signaling: Its effect on the visual cliff behavior of 1-year-olds. Developmental Psychology, 21, 195200. http://psycnet.apa.org/doi/10.1037/0012-1649.21.1.195

Striano, T., \& Reid, V.M. (2006). Social cognition in the first year. Trends in Cognitive Sciences, 
10, 471-476. DOI:10.1016/j.tics.2006.08.006

Thelen, E., \& Spencer, J.P. (1998). Postural control during reaching in young infants: A dynamic systems approach. Neuroscience and Biobehavioral Reviews, 22, 507-514. DOI:

10.1016/S0149-7634(97)00037-7

Vaish, A., Grossmann, T., \& Woodward, A. (2008). Not all emotions are created equal: The negativity bias in social-emotional development. Psychological Bulletin, 134, 383-403. DOI: $10.1037 / 0033-2909.134 .3 .383$

Van der Schalk, J., Hawk, S. T., Fischer, A. H., \& Doosje, B. J. (2011). Moving faces, looking places: The Amsterdam Dynamic Facial Expressions Set (ADFES). Emotion, 11, $907-$ 920. DOI: $10.1037 / \mathrm{a} 0023853$

Walker-Andrews, A.S. (1997). Infants' perception of expressive behaviors: Differentiation of multimodal information. Psychological Bulletin, 121, 437-456. DOI: 10.1037/00332909.121.3.437

Wilcox, B.M., \& Clayton, F.L. (1968). Infant visual fixation on motion pictures of the human face. Journal of Experimental Child Psychology, 6, 22-32. DOI: 10.1016/00220965(68)90068-4 